\title{
Data driven generation of Purkinje cell spike train correlations to study input output relations in deep cerebellar nuclei neurons
}

\author{
Selva K Maran*, Dieter Jaeger \\ From Nineteenth Annual Computational Neuroscience Meeting: CNS*2010 \\ San Antonio, TX, USA. 24-30 July 2010
}

Cerebellar output generation in the deep cerebellar nuclei (DCN) presents a fundamental but poorly understood component of cerebellar function. Experimental data obtained in vitro and in anesthetized animals as well as modeling studies have shown that correlations between Purkinje cell inputs are important in determining the spike rate and spike pattern of DCN output. How a DCN neuron process the synaptic inputs from hundreds of presynaptic Purkinje cells in an awake behaving animal, and the importance of the correlation structure between those inputs, remain unknown, however. We aim to quantitatively assess the correlation structure of Purkinje cells from data recorded in awake mice, and to determine how such correlations interact with the intrinsic DCN physiological properties.

It is experimentally impossible to record all the inputs that are reaching a single DCN neuron in an awake animal. Therefore methods need to be developed that can extrapolate the correlations found in a small number of simultaneous recordings to an ensemble of several hundred artificial spike trains with the same correlation structure. Several analytical methods have been developed to generate sets of spike trains with specific correlations in spike times as well as spike rates [1-4]. On the other hand, experimental evidence from Purkinje cell recordings in anesthetized animals point to specific correlation features such as shared pauses [5], synchronous single spikes [6], and synchronous climbing fiber bursts [7]. We will analyze the spike train data obtained from multi-electrode recordings in awake behaving mice to determine the presence of correlation features as well as spike rate correlations. We will derive artificial spike

\footnotetext{
* Correspondence: sseland@emory.edu

Department of Biology Emory University, Atlanta, Georgia, 30329, USA
}

trains using a method based on previous algorithms [1-4]. We will also introduce feature based specific correlations in our constructed artificial spike trains present in our recordings. We will optimize this process so that artificial spike trains show the same inter-spike interval statistics, rate correlations, and feature based correlations seen in recorded spike trains. These data will allow us to drive a multicompartmental DCN model neuron to study synaptic integration in the awake condition. Because large sets of correlated spike trains are required for all single neuron model explorations of signal processing in awake animals we expect that the generation of realistic input correlations will become an important topic as the field of simulating neural properties in vitro matures to simulating the awake condition.

\section{Published: 20 July 2010}

\section{References}

1. Niebur E: Generation of synthetic spike trains with defined pairwise correlations. Neural Comput 2007, 19(7):1720-38.

2. Macke $J H$, Berens $P$, Ecker AS, Tolias AS, Bethge M: Generating spike trains with specified correlation coefficients. Neural Comput 2009, 21(2):397-423.

3. Krumin M, Shoham S: Generation of spike trains with controlled autoand cross-correlation functions. Neural Comput 2009, 21(6):1642-64.

4. Brette R: Generation of correlated spike trains. Neural Comput 2009, 21(1):188-215.

5. Gauck $V$, Jaeger D: The control of rate and timing of spikes in the deep cerebellar nuclei by inhibition. J Neurosci 2000, 20(8):3006-3016.

6. Shin SL, Hoebeek FE, Schonewille M, De Zeeuw Cl, Aertsen A, De Schutter E: Regular patterns in cerebellar Purkinje cell simple spike trains. PLoS One 2007, 2(5):e485.

7. Heck D H, Thach W T, et al: On-beam synchrony in the cerebellum as the mechanism for the timing and coordination of movement. Proc Natl Acad Sci USA 2007, 104(18):7658-7663.

doi:10.1186/1471-2202-11-S1-P116

Cite this article as: Maran and Jaeger: Data driven generation of

Purkinje cell spike train correlations to study input output relations in deep cerebellar nuclei neurons. BMC Neuroscience 2010 11(Suppl 1):P116. 